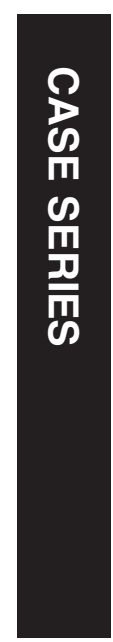

\section{'Toy' laser macular burns in children: 12-month update}

\begin{abstract}
Purpose There is increasing evidence that high-powered hand-held laser devices cause retinal injury. We present 12-month follow-up data for three patients that we previously reported with such retinal injuries. Methods A retrospective case series of three children with maculopathy secondary to exposure to high-power hand-held laser devices. All children underwent clinical examination and spectral domain optical coherence tomography (SD-OCT) at presentation and follow-up. Funduscontrolled microperimetry was also undertaken 12-19 months after exposure. Results Three children sustained macular injury after exposure to a high-powered handheld laser. Acutely, they presented with a 'vitelliform-like' maculopathy with reduced vision. Over the course of follow-up, the best corrected Snellen acuity in all three patients improved to 'normal' levels (range 6/6-6/9). Long-term deficits in foveal retinal sensitivity were identified in two patients using funduscontrolled microperimetry. SD-OCT imaging showed persistent disruption of the foveal outer photoreceptor layers in all three children. Conclusion Although visual acuity improved over time, deficits in microperimetry and SDOCT persisted. All three children had retinal pigment epithelium changes, requiring follow-up for longer-term sequelae of laser injuries such as expansion of retinal atrophy and development of choroidal neovascular membranes.
\end{abstract}

Eye (2016) 30, 492-496; doi:10.1038/eye.2015.222; published online 27 November 2015

, Glossop Road

Sheffield, South Yorkshire

S10 2JF, UK

Tel: +44 (0)11 42713281 ;

Fax: +44 (0)1142713682.

E-mail: fahd.quhill@sth.nhs.uk

Received: 16 May 2015 Accepted in revised form: 22 September 2015

Published online:

27 November 2015
There is mounting evidence to suggest that highpowered, hand-held laser devices purchased via online retailers have the capacity to cause retinal injury. In 2014, we published a case series of five
N Raoof ${ }^{1}$, J O'Hagan², N Pawlowska ${ }^{3}$ and F Quhill ${ }^{3}$

children with visually significant macular injury following exposure to such devices. ${ }^{1}$ Since publication there have been 7 other reports detailing 24 individuals, predominantly children, who had suffered similar retinal injuries. ${ }^{2-8}$ The long-term prognosis of such injuries remains unclear. In this paper, we present a 12-month update for three children from our earlier case series.

\section{Case 1}

A 9-year-old boy presented to us $24 \mathrm{~h}$ following a left macular laser burn from a hand-held laser device purchased online. His left corrected Snellen vision was 6/15. An acute vitelliform-like maculopathy was present in the left eye (Figure 1a). Spectral domain optical coherence tomography (SD-OCT) imaging was undertaken (Figure 1b). Within 3 days the retinal opacification had resolved with laser burns visible clinically at the left macula. Examination of the laser devices revealed three separate devices (ranging from $42-72 \mathrm{~mW}$ in power). ${ }^{1}$ It is not clear which of the devices caused the retinal injury.

Two months following the injury, visual acuity had improved to $6 / 9$ in the affected eye, improving to $6 / 6$ at 19 months post injury. At 19 months, microperimetry (using a Nidek MP-1 microperimeter, set with Goldmann stimulus size III at a mesopic background luminance of $1.27 \mathrm{~cd} / \mathrm{m}^{2}$ ) revealed reduced foveal sensitivity of around $-8 \mathrm{~dB}$ at fixation (Figure 1c), associated with reduced contrast sensitivity of 1.5 at $1 \mathrm{~m}$ in the left eye (Pelli-Robson chart). ${ }^{9}$ Repeat SD-OCT imaging revealed persistent outer retinal changes (Figure 1d).

\section{Case 2}

An 11-year-old boy was referred from a local optometrist with decreased vision (6/7.5 in both eyes) and bilateral yellow macular lesions. The child admitted misusing a laser device; the laser device could not be obtained for inspection. His vision deteriorated 8 weeks later in clinic (6/12 right eye, 6/15 left eye). SD-OCT acquired by the 

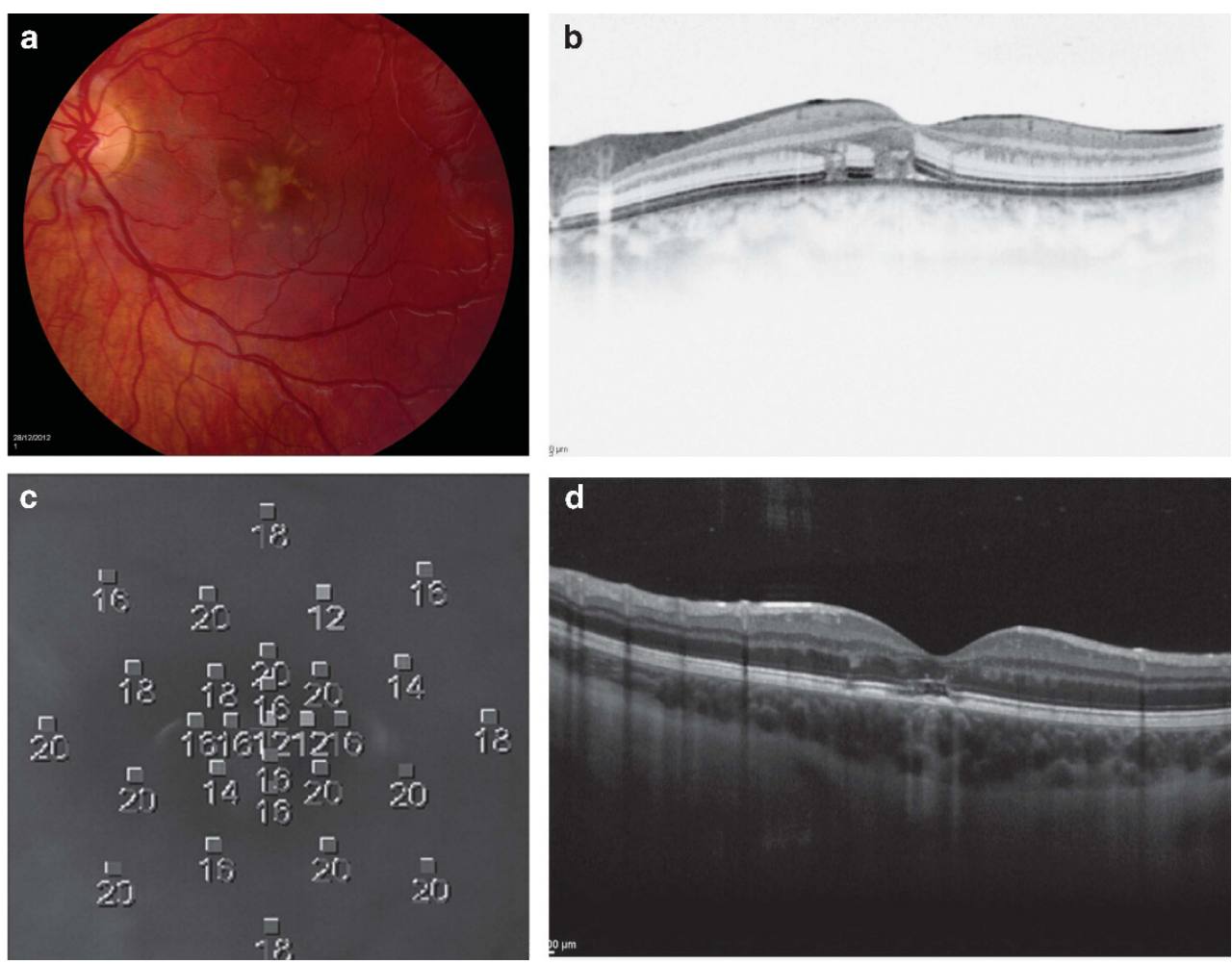

Figure 1 Colour photograph of the left eye (a) at presentation revealing a yellow vitelliform-like lesion representing the acute laser injury. A SD-OCT (b) acquired at presentation revealed two focal hyper-reflective columns at the fovea extending from the retinal pigment epithelium (RPE), involving all outer retinal layers, terminating at the outer plexiform layer, with opacification of Henle's fibre layer. At 19 months post injury (c) microperimetry revealed the presence of reduced foveal sensitivity of around $-8 \mathrm{~dB}$ at fixation with a repeat SD-OCT showing persistent outer retinal changes (d) with thinning of RPE, interruption of the interdigitation/ellipsoid bands and irregularity of the external limiting membrane at the fovea. Pinpoint hyper reflective changes were also evident in the outer nuclear layer.

optometrist at presentation is shown (Figures 2a and b). Over a 12-month follow-up period, his vision improved to $6 / 9$ in each eye, although there was a persistent hyporeflective cavity extending from the interdigitation zone to the external limiting membrane at the fovea in the right eye and more subtle disruption in the same region of the left eye. Microperimetry once again revealed subtle deficits of between -4 and $-8 \mathrm{~dB}$ in both eyes (Figures $2 \mathrm{c}$ and $\mathrm{d}$ ).

\section{Case 3}

An 8-year-old boy was incidentally noted to have reduced right vision of $6 / 12$, after attending the emergency department with a minor head injury. The child admitted playing with a hand-held laser device several months earlier. Examination revealed right foveal RPE changes, consistent with laser burns. SD-OCT revealed disruption of the outer retinal bands with pigment migration in the right eye at the fovea (Figure 3a). At 12 months, his vision improved to $6 / 7.5$ in the right eye, with persistent subtle disruption of the photoreceptor layer on OCT (Figure 3b). Microperimetry revealed normal foveal sensitivity (Figure 3c).

\section{Discussion}

All three patients in this study showed improvement in their visual acuity, to at least 6/9. Persistent defects remained, both clinically and on SD-OCT imaging, however. Microperimetry revealed decreased foveal sensitivity in two children, suggesting a functional effect on vision; longer follow-up is required to monitor expansion of foveal scarring over time, as well as development of choroidal neovascular membranes (observed in another child at presentation ${ }^{1}$ ). The third child had persistent subtle outer retinal defects affecting the outer photoreceptor layer but no decrease in foveal sensitivity evident on microperimetry. We postulate this is due to greater than normal foveal sensitivity in this child before injury, although we expect a decline in sensitivity over time due to injury.

We believe such laser injuries are an emerging phenomenon. Between December 2013 and December 2014, there were seven reports of similar injuries (24 patients). ${ }^{2-8}$ In the 12 months before, only 2 such injuries were described. ${ }^{10,11}$ 


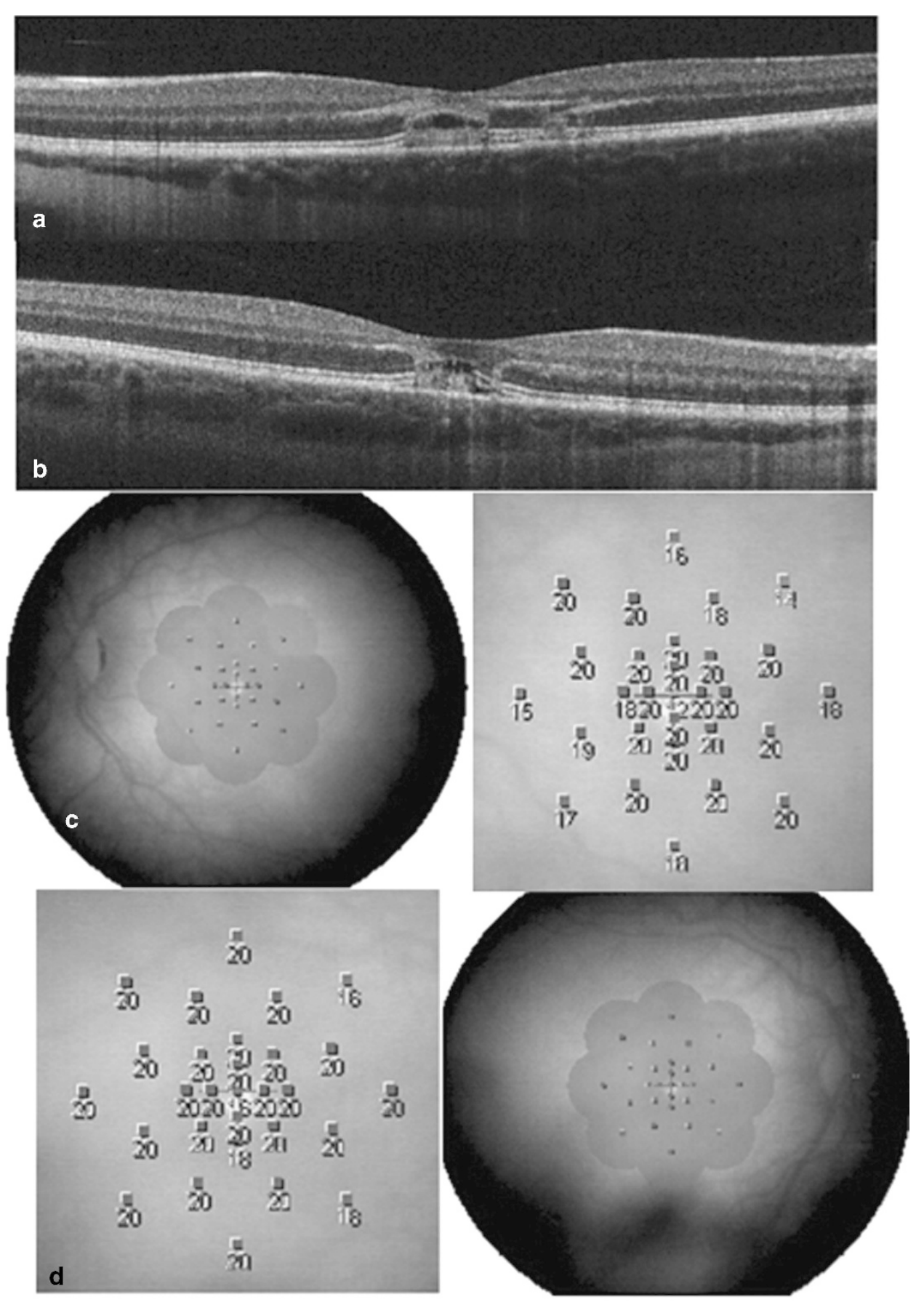

Figure 2 SD-OCT imaging, acquired by the optometrist at presentation $(a, b)$, revealed disruption and hyper-reflectivity in the outer retinal layers involving the interdigitation and ellipsoid zones at both fovea extending towards the outer plexiform layer. At 12 months follow-up microperimetry reveals subtle deficits of between -4 and $-8 \mathrm{~dB}$ in both eyes $(\mathrm{c}, \mathrm{d})$.

With improvement in technology, hand-held laser devices are becoming increasingly powerful and available via online marketplaces, often not conforming to EU or British Safety Standards (BS EN 60825-1:2014), making the policing of such devices difficult. We advise that laser devices, whether they are 'toys' or intended as laser pointers, are only purchased from sources where laser safety specifications are unquestionably being upheld, for example, UK high street retailers. We encourage any ophthalmologist who encounters an ocular injury secondary to a laser device to inform local trading standards. Finally, we call on the UK Government to fund a public health campaign to prevent further consumers becoming patients. 


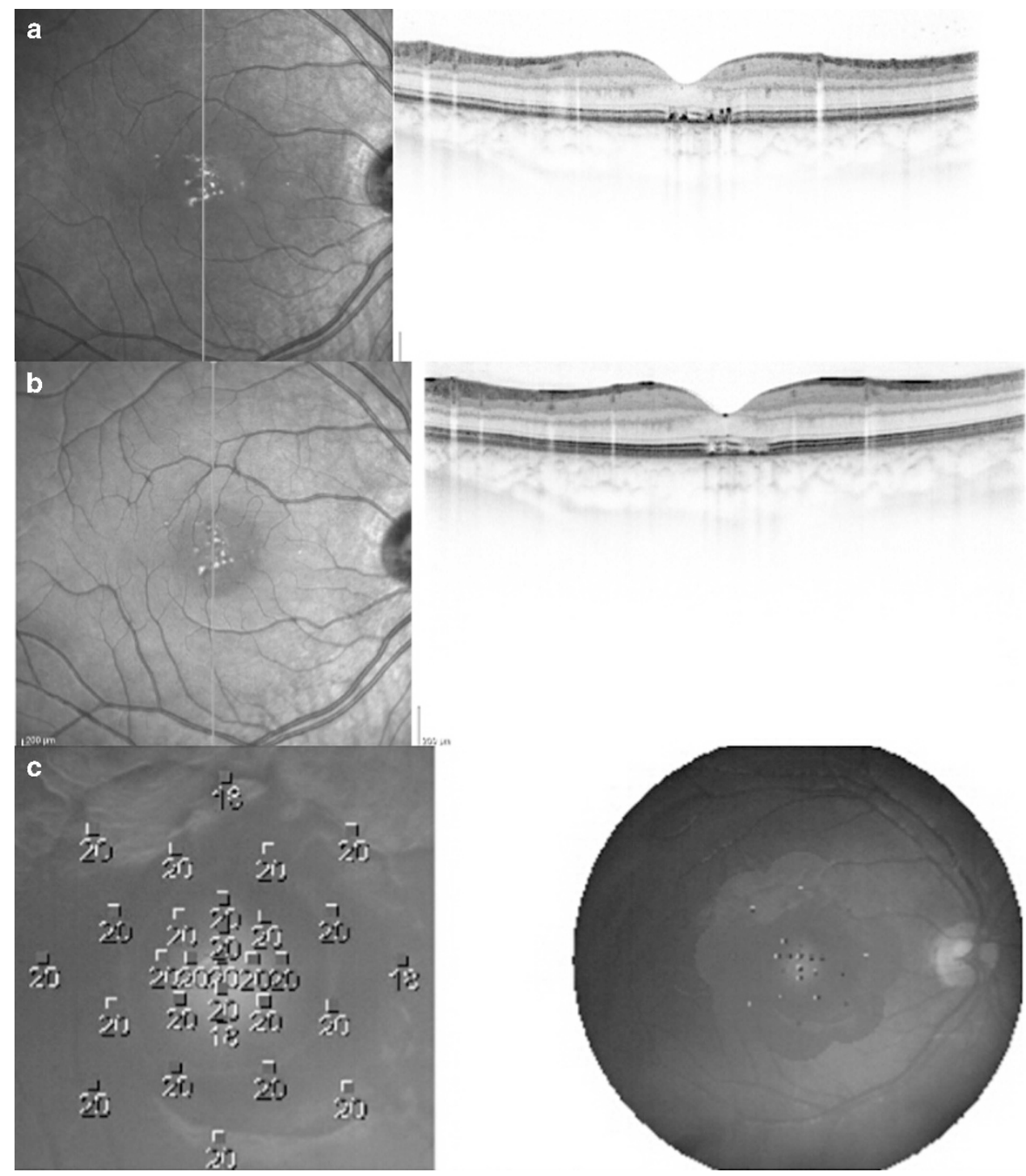

Figure 3 SD-OCT imaging at baseline revealed disruption of the outer retinal bands with RPE migration in the right eye at the fovea (a). At 12-month follow-up, the vision had improved to 6/7.5 in the right eye, with persistent, although more subtle disruption of the photoreceptor layer on OCT (b). Microperimetry revealed normal foveal sensitivity (c).

\section{Summary}

\section{What was known before}

- Hand-held lasers with the potential to cause retinal injuries can be obtained by the general public. Retinal injuries from such laser devices can result in acute vision loss.

\section{What this study adds}

- Vision can recover to 'normal' levels following retinal injuries from hand-held laser devices. Defects in SD-OCT imaging and microperimetry, persisting after 12 months follow-up, demonstrate that deficits in visual function can remain, necessitating follow-up for sequela of laser injuries.

\section{Conflict of interest}

The authors declare no conflict of interest.

\section{References}

1 Raoof N, Chan TK, Rogers NK, Abdullah W, Haq I, Kelly SP et al. Toy laser macular burns in children. Eye (Lond) 2014; 28(2): 231-4.

2 Alsulaiman SM, Alrushood AA, Almasaud J, Alzaaidi S, Alzahrani Y, Arevalo JF et al. King Khaled Eye Specialist Hospital Collaborative Retina Study Group. High-power handheld blue laser-induced maculopathy: the results of the King Khaled Eye Specialist Hospital Collaborative Retina Study Group. Ophthalmology 2014; 121(2): 566-572.

3 Yiu G, Itty S, Toth CA. Ocular safety of recreational lasers. JAMA Ophthalmol 2014; 132(3): 245-246. 
4 Dhoot DS, Xu D, Srivastava S. High-powered laser pointer injury resulting in macular hole formation. J Pediatr 2014; 164 (3): 668.

5 Petrou P, Patwary S, Banerjee PJ, Kirkby GR. Bilateral macular hole from a handheld laser pointer. Lancet 2014; 383: 1780.

6 Keunen JE, Delbecq AL, Cruysberg JR, van Meurs JC, Gan IM, Berendschot TT. [Laser pointers are not toys; eye injury with permanent loss of visual acuity]. Ned Tijdschr Geneeskd 2014; 158: A7813.

7 Lee GD, Baumal CR, Lally D, Pitcher JD, Vander J, Duker JS. Retinal injury after inadvertent handheld laser exposure. Retina 2014; 34(12): 2388-2396.
8 Lim ME, Suelzer J, Moorthy RS, Vemuri G. Thermal macular injury from a $154 \mathrm{~mW}$ green laser pointer. J AAPOS 2014; 18 (6): 612-614.

9 Maija Mantyjarvi MD, Tarja Laitinen MD. Normal values for the Pelli-Robson contrast sensitivity test. J Cataract Refract Surg 2001; 27(2): 261-266.

10 Dirani A, Chelala E, Fadlallah A et al. Bilateral macular injury from a green laser pointer. Clin Ophthalmol 2013; 7: 2127-2130.

11 Rusu I, Sherman J, Gallego-Pinazo R et al. Spectral-domain optical coherence tomography and fundus autofluorescence findings in a case of laser pointer-induced maculopathy. Retin Cases Brief Rep 2013; 7(4): 371-375. 